\title{
Plasma levels of matrix metalloproteinase-2, $-3,-10$, and tissue inhibitor of metalloproteinase- 1 are associated with vascular complications in patients with type 1 diabetes: the EURODIAB Prospective Complications Study
}

Stijn A Peeters ${ }^{1,2}$, Lian Engelen ${ }^{1}$, Jacqueline Buijs², Nish Chaturvedi ${ }^{3}$, John H Fuller ${ }^{4}$, Casper G Schalkwijk ${ }^{1 \dagger}$, Coen D Stehouwer ${ }^{1 *}$ and EURODIAB Prospective Complications Study Group

\begin{abstract}
Background: Impaired regulation of extracellular matrix remodeling by matrix metalloproteinases (MMPs) and tissue inhibitor of metalloproteinase (TIMP) may contribute to vascular complications in patients with type 1 diabetes. We investigated associations between plasma MMP-1, $-2,-3,-9,-10$ and TIMP-1, and cardiovascular disease (CVD) or microvascular complications in type 1 diabetic patients. We also evaluated to which extent these associations could be explained by low-grade inflammation (LGl) or endothelial dysfunction (ED).

Methods: 493 type 1 diabetes patients (39.5 \pm 9.9 years old, 51\% men) from the EURODIAB Prospective Complications Study were included. Linear regression analysis was applied to investigate differences in plasma levels of MMP-1, $-2,-3,-9,-10$, and TIMP-1 between patients with and without CVD, albuminuria or retinopathy. All analyses were adjusted for age, sex, duration of diabetes, Hba1c and additionally for other cardiovascular risk factors including LGl and ED.

Results: Patients with CVD ( $n=118)$ showed significantly higher levels of TIMP-1 $[\beta=0.32$ SD (95\%Cl: 0.12; 0.52)], but not of MMPs, than patients without CVD $(n=375)$. Higher plasma levels of MMP-2, MMP-3, MMP-10 and TIMP-1 were associated with higher levels of albuminuria ( $p$-trends were $0.028,0.004,0.005$ and 0.001 , respectively). Severity of retinopathy was significantly associated with higher levels of MMP-2 ( $p$-trend $=0.017$ ). These associations remained significant after further adjustment for markers of LGI and ED.
\end{abstract}

Conclusions: These data support the hypothesis that impaired regulation of matrix remodeling by actions of MMP-2, -3 and-10 and TIMP-1 contributes to the pathogenesis of vascular complications in type 1 diabetes.

Keywords: Type 1 diabetes, Matrix metalloproteinase, Tissue inhibitor of metalloproteinase, Cardiovascular disease, Albuminuria, Retinopathy

\footnotetext{
* Correspondence: cda.stehouwer@mumc.nl

${ }^{\dagger}$ Equal contributors

${ }^{1}$ Department of Internal Medicine, Maastricht University Medical Centre

P.O. Box 5800, 6202 AZ, Maastricht, the Netherlands

Full list of author information is available at the end of the article
} 


\section{Background}

Type 1 diabetes is associated with an increased risk of macro- and microvascular disease [1,2]. The exact mechanisms leading to vascular damage in type 1 diabetes have not been fully established [3], but impaired regulation of extracellular matrix (ECM) remodeling by matrix metalloproteinases (MMPs) may contribute to the development of vascular complications [4,5]. Increased MMP activity has been associated with increased matrix turnover and potentially to pathological reorganization of the ECM in atherosclerosis, aneurysm formation, plaque disruption [4] and, in diabetic nephropathy, to glomerular basement membrane thickening [5].

MMPs are a group of zinc- and calcium-dependent endopeptidases, which degrade and rebuild proteins of the ECM, such as collagen, elastin, gelatin and casein [6]. Thus far, more than 20 different MMPs have been identified, which, according to their various functions, can be divided into collagenases (e.g. MMP-1), gelatinases (MMP-2 and MMP-9), stromelysines (e.g. MMP-3 and MMP-10), matrilysines, and others. These enzymes can be excreted by various cells (e.g. fibroblasts, endothelial cells, monocytes and macrophages) or can be incorporated in the cellular membrane. Tissue inhibitors of metalloproteinases (TIMP-1-4) and $\alpha 2$-macroglobulin inhibit the action of MMPs [6].

Plasma levels of MMPs have been observed to be higher in individuals with type 1 diabetes, compared to controls [7-9]. The association between circulating MMPs and macrovascular disease in patients with type 1 diabetes has not been investigated, while studies on the associations between MMP levels and microvascular complications seem contradictory. For example, high levels of serum MMP-9 and a high MMP-9-to-TIMP ratio have been related to retinopathy [10], and high levels of plasma MMP-10 have been associated with nephropathy and proliferative retinopathy [8]. In contrast, other studies did not show significant associations between plasma levels of MMP-2, -9 or TIMP-1 and microvascular complications [7,11]. In fact, most studies were rather small [7,9-11] or limited to only one or two MMPs [7-11]. In one large study of 269 type 1 diabetes patients and 269 non-diabetic controls [8], plasma MMP-10 was associated with nephropathy and proliferative retinopathy. However, plasma TIMP was not measured, whereas this may be of importance as MMP-10 activity is dependent on the amount of inhibitors. Nevertheless, in type 1 diabetes, hyperglycaemia, low-grade inflammation (LGI) and endothelial dysfunction (ED) have been found to be associated with higher plasma and tissue levels of MMPs and TIMP [5,9,12], and LGI and ED are consistently associated with macro- and microvascular complications [13-15].

In view of these considerations, we hypothesized that high plasma levels of MMPs and TIMP are related to macro- and microvascular complications in individuals with type 1 diabetes, possibly through associations with LGI and ED. We investigated these hypotheses in a cross-sectional study of patients with type 1 diabetes, in whom we assessed macro- and microvascular disease and measured plasma levels of MMP-1, -2, -3, -9, -10 and TIMP-1, as well as biomarkers of LGI and ED.

\section{Methods}

\section{Study population}

In this analysis, data were used from the EURODIAB Prospective Complications Study, a European prospective cohort study that has been described in detail previously [16,17].

In brief, baseline inclusion was performed between 1989 and 1991 in 3,250 patients with type 1 diabetes. Inclusion criteria were defined as a clinical diagnosis of type 1 diabetes before the age of 36 years, and requirement of continuous insulin therapy within the first year of diagnosis. Patients aged between 15 and 60 years were recruited from 31 centres in 16 European countries. Sample selection was stratified by sex, age group and duration of diabetes to ensure sufficient representation of all categories. These patients were invited for a follow-up visit 7-9 years after baseline enrolment. Of the 3,250 included patients, 1,880 (57.8\%) returned for re-examination. At follow-up, a cross-sectional nested case-control study was performed in a subset of patients $(n=543)$. Cases $(n=348)$ were those with one or more complications and controls $(n=195)$ were those with no evidence of complications [18]. This type 1 diabetic patient control group was used to investigate differences in plasma levels of MMPs and TIMP-1 compared with type 1 diabetic patients with vascular complications. For the current analyses, clinical data, plasma samples for analysis of MMPs and TIMP-1 and information about potential confounders and mediators were available in 493 of these 543 patients. The study was approved by the local Ethics Committee at each centre and all patients gave informed consent.

\section{Main determinants}

Concentrations of MMPs and TIMP-1 were determined in plasma samples that were stored at $-80^{\circ} \mathrm{C}$ after collection and in our laboratory until analyses and they were never thawed previously. Plasma levels of MMP-1, MMP-2, MMP-3, MMP-9, MMP-10 and TIMP-1 were measured using a commercially available enzyme-linked immunosorbent assay (ELISA) kit [Human MMP 3-Plex Kit (for MMP-1, -3 and -9), Human MMP-2-Plex Kit (for MMP-2 and -10) and Human TIMP-1 Kit, MSD, Rockville, United States of America] according to the manufacturer's protocol. Plasma samples of 10, 25 and 
2.5 microliter were used for the MMP-3-plex kit, MMP2-plex kit and TIMP-1 kit, respectively. The MMPs were detected in both a pro- and an active form. TIMP-1 was detected only in the active form. The intra- and interassay coefficients of variation were $7.0 \%$ and $8.0 \%$ for MMP-1, 4.5\% and 5.9\% for MMP-2, 8.4\% and 12.3\% for MMP-3, 5.3\% and 8.9\% for MMP-9, 4.4\% and 9.7\% for MMP-10, and $4.3 \%$ and 5.2\%for TIMP-1, respectively.

\section{Main outcomes}

\section{Macrovascular disease}

Cardiovascular disease (CVD) was defined as a cardiovascular event in a patient's medical history, including myocardial infarction, angina, coronary artery bypass graft, stroke or ischaemic changes in a centrally Minnesota-coded ECG [19].

\section{Microvascular disease}

Albumin excretion rates were measured from duplicate 24-hour urine collections [17]. Micro- and macroalbuminuria were defined as an albumin excretion rate between 20 and $200 \mu \mathrm{g} / \mathrm{min}$, or above $200 \mu \mathrm{g} / \mathrm{min}$, respectively. We also estimated the glomerular filtration rate (eGFR) using the Chronic Kidney Disease Epidemiology Collaboration (CKD-EPI) equation [20].

Retinopathy was assessed from retinal photographs according to the EURODIAB protocol; non-proliferative retinopathy was defined as the presence of one or more microaneurysms, hemorrhages, and/or hard exudates. Proliferative retinopathy was defined as presence of any new vessels, fibrous proliferations, pre-retinal haemorrhages, vitreous hemorrhages or photocoagulation scars [21].

\section{Other variables}

Apart from demographic data, additional information about medication, smoking history, systolic blood pressure and duration of diabetes was collected. Body weight and height were measured in patients wearing indoor clothing without shoes. With these values body mass index (BMI) was calculated. Fasting blood samples were taken for measurements of lipid profile and glycemic control. Cholesterol and triglyceride levels were measured by enzymatic colorimetric tests [22], HDL was measured directly [23]. Friedewald's formula was used to calculate LDL levels [24]. Glycated hemoglobin (HbA1c) was measured by a latex-enhanced turbidimetric immunoassay (Roche Products, Welwyn Garden City, UK). The reference range for this assay was $4.2-6.2 \%$.

\section{Markers of LGI}

Plasma levels of C-reactive protein (CRP) were measured with a highly sensitive ELISA, developed and validated in our own laboratory [18]. Plasma levels of interleukin6 (IL-6) and tumor necrosis factor- $\alpha$ (TNF- $\alpha$ ) were measured using commercially available ELISA kits (R\&D Systems, Oxon, U.K.) [18]. Intra- and inter-assay coefficients of variation were 3.9 and $8.7 \%$ for CRP, 4.5 and 9.0\% for IL-6, and 7.3 and $8.5 \%$ for TNF- $\alpha$, respectively.

\section{Markers of ED}

Plasma soluble E-selectin (sE-selectin) and soluble vascular cell adhesion molecule-1 (sVCAM-1) were measured by sandwich enzyme immunoassays (R\&D Systems, OXON, U.K.) in duplicate. Individual mean values of the duplicates were used in the ED scores. Intra- and interassay coefficients of variation were $2.1 \%$ and $3.1 \%$ for sEselectin, and $4.0 \%$ and $9.1 \%$ for sVCAM-1, respectively.

\section{Statistical analyses}

All analyses were performed using the Statistical Package for Social Sciences (SPSS), version 20 (IBM Corporation, Armonk, NY, USA). Log transformation was performed for variables with a skewed distribution (triglycerides, serum creatinine, eGFR, MMP-1, MMP-2, MMP-3, MMP-9, MMP-10, CRP, IL-6 and TNF- $\alpha$ ). Student's $\mathrm{t}$ - or Chi-Square tests were performed for comparisons of characteristics between individuals with and without vascular complications, as appropriate.

Linear regression analyses were performed to examine the extent to which plasma levels of MMP-1, MMP-2, MMP-3, MMP-9, MMP-10 and TIMP-1 differed between individuals with and without CVD or microvascular complications as well as to test for linear trends in plasma levels of MMPs and TIMP-1 between both micro- and macroalbuminuria and between non-proliferative and proliferative retinopathy. Results of these analyses are presented as standardized regression coefficients to enable comparison of the magnitude of the associations. We have only investigated single MMPs and TIMP-1 in the analyses and have not adjusted for the presence of others, because each MMP has its own substrate specificity and actions.

Analyses were adjusted for age, sex, duration of diabetes and HbA1c (model 1). Further adjustments were made for LDL, HDL, systolic blood pressure, eGFR, antihypertensive medication, triglycerides, BMI and smoking, and for the presence of CVD, albuminuria and/or retinopathy, as appropriate (model 2). Markers of ED (model 3) and LGI (model 4) were subsequently added to this model to explore whether the association between plasma levels of MMPs and TIMP-1 on the one hand and CVD and microvascular complications on the other (if any) were explained (i.e., potentially mediated) by LGI and/or ED. Markers of LGI were comprised into an overall LGI z-score by computing a z-score after averaging the z-scores of lnCRP, $\operatorname{lnIL}-6$ and $\operatorname{lnTNF}-\alpha$. ED z-scores were expressed after averaging of the z-scores of sVCAM-1 and sE-selectin. A $\mathrm{z}$-score represents the difference between the individual biomarker score and the mean value in the population, 
expressed in units of the standard deviation of the study population. This enables comparison of markers expressed in different units. The two scores represent a more robust value of the individual's levels of LGI and ED, as they reduce the influence of biological variability expected when LGI and ED should be characterized by levels of each biomarker separately [25].

\section{Results}

\section{Patients' characteristics}

Table 1 shows the characteristics of patients with or without vascular complications. Patients with vascular complications were characterized by higher age, BMI and systolic blood pressure. Levels of HbA1c, LDL, triglycerides, MMP-1, MMP-2, MMP-3, MMP-9, MMP-10, TIMP-1 and markers of LGI and ED were significantly higher in individuals with vascular complications compared to those without.

\section{Associations between MMPs, TIMP-1 and cardiovascular disease}

Significantly higher plasma levels of TIMP- $1[\beta=0.27$ SD $(95 \% \mathrm{CI}: 0.06 ; 0.48)]$ were observed in individuals with CVD $(n=118)$ as compared to those without, after

Table 1 Clinical characteristics of the study population

\begin{tabular}{|c|c|c|c|}
\hline & $\begin{array}{l}\text { Vascular complications } \\
(\mathrm{n}=306)\end{array}$ & $\begin{array}{l}\text { No vascular complications } \\
(n=187)\end{array}$ & p-value \\
\hline Age (years) & $41.6(10.4)$ & $36.1(8.1)$ & $<0.001$ \\
\hline Sex (male/female, \%) & $54 / 46$ & $46 / 54$ & 0.096 \\
\hline BMI $\left(\mathrm{kg} / \mathrm{m}^{2}\right)$ & $24.8(3.5)$ & $23.9(2.6)$ & $<0.001$ \\
\hline HbA1c (\%) & $9.0(1.6)$ & $7.7(1.3)$ & $<0.001$ \\
\hline $\mathrm{HbA} 1 \mathrm{c}(\mathrm{mmol} / \mathrm{mol})$ & $75(17.4)$ & $61(14.1)$ & $<0.001$ \\
\hline Duration of diabetes (years) & $25.2(8.9)$ & $15.5(7.0)$ & $<0.001$ \\
\hline LDL cholesterol (mmol/l) & $3.30(1.06)$ & $2.88(0.93)$ & $<0.001$ \\
\hline HDL cholesterol (mmol/l) & $1.59(0.42)$ & $1.68(0.45)$ & 0.032 \\
\hline Triglycerides (mmol/l) & $1.13[0.84-1.58]$ & 0.85 [0.67-1.09] & $<0.001$ \\
\hline Smoking (no/former/current) (\%) & $36 / 31 / 33$ & $46 / 26 / 28$ & 0.047 \\
\hline Systolic blood pressure (mmHg) & $127(21)$ & $115(13)$ & $<0.001$ \\
\hline Diastolic blood pressure $(\mathrm{mmHg})$ & $75(12)$ & $73(10)$ & 0.085 \\
\hline Serum creatinine ( $\mu \mathrm{mol} / \mathrm{l})$ & $76.0[68.0-90.0]$ & $71.0[64.0-79.0]$ & $<0.001$ \\
\hline eGFR $\left(\mathrm{ml} / \mathrm{min} / 1.73 \mathrm{~m}^{2}\right)$ & $95.7[79.0-107.9]$ & $107.9[97.2-115.6]$ & $<0.001$ \\
\hline Cardiovascular disease (\%) & 38.6 & - & - \\
\hline Albuminuria (normo-/micro-/macro-)(\%) & $37.9 / 25.2 / 36.9$ & - & - \\
\hline Retinopathy (no/background/proliferative) (\%) & 11.4/40.9/47.7 & - & - \\
\hline Antihypertensive medication (\%) & 45.4 & 5.9 & $<0.001$ \\
\hline ACE-inhibitor (\%) & 37.6 & 4.3 & $<0.001$ \\
\hline MMP-1 (ng/ml) & $12.8[7.0-19.9]$ & $10.8[5.9-16.5]$ & 0.006 \\
\hline MMP-2 (ng/ml) & 110 [100-120] & 103 [96-110] & $<0.001$ \\
\hline MMP-3 (ng/ml) & $17.8[11.2-28.3]$ & $12.9[8.3-19.6]$ & $<0.001$ \\
\hline MMP-9 (ng/ml) & $122[81-195]$ & $110[66-172]$ & 0.023 \\
\hline MMP-10 (pg/ml) & 1285 [942-1931] & $1077[790-1635]$ & $<0.001$ \\
\hline TIMP-1 (ng/ml) & $313(100)$ & $256(74)$ & $<0.001$ \\
\hline C-reactive protein (mg/l) & $1.28[0.46-2.68]$ & $0.71[0.35-1.80]$ & $<0.001$ \\
\hline Interleukin-6 (pg/ml) & $2.12[1.35-3.86]$ & $1.57[1.06-2.50]$ & $<0.001$ \\
\hline Tumor necrosis factor-a $(\mathrm{pg} / \mathrm{ml})$ & $3.16[2.33-4.42]$ & $2.22[1.68-2.85]$ & $<0.001$ \\
\hline Soluble e-selectin (ng/ml) & $35.7(16.6)$ & $31.1(11.1)$ & $<0.001$ \\
\hline Soluble vascular cell adhesion molecule-1 (ng/ml) & $435(144)$ & $378(104)$ & $<0.001$ \\
\hline
\end{tabular}

Data are presented as means (standard deviation), median [inter-quartile range], or percentages, as appropriate.

Vascular complication: presence of previous CVD, macroalbuminuria, or proliferative retinopathy or the combination of microalbuminuria and non-proliferative retinopathy; BMI, body mass index; HbA1c, glycated hemoglobin; LDL, low-density lipoprotein; HDL, high-density lipoprotein; eGFR, estimated glomerular filtration rate by CKD-EPI formula; MMP, matrix metalloproteinase; TIMP-1, tissue inhibitor of metalloproteinase-1. 
adjustment for age, sex, duration of diabetes and HbA1c (Additional file 1: Table S1, Model 1). The association became even stronger $[0.32(0.12 ; 0.52)]$ after further adjustment for other cardiovascular risk factors, albuminuria and retinopathy (Figure 1; Additional file 1: Table S1, Model 2). In contrast, MMP-1, MMP-2, MMP-3, MMP-9 and MMP-10 did not differ between groups.

\section{Associations between MMPs, TIMP-1 and albuminuria}

Patients with microalbuminuria $(n=77)$ showed significantly higher plasma levels of MMP-2 compared to patients with normal urinary albumin excretion after adjustment for cardiovascular risk factors and other vascular complications [0.32 (0.03; 0.61)] (Figure 2A; Additional file 2: Table S2, Model 2). Macroalbuminuria $(n=113)$, compared to normoalbuminuria, was associated with even higher levels of MMP-2 [0.38 (0.10; 0.66)] (Figure 2A; Additional file 2: Table S2). In this group, additionally, higher plasma levels of MMP-3 [0.33 (0.11; 0.54)], MMP-10 [0.38 (0.08; 0.67)] and TIMP-1 [0.45 (0.18; $0.71)$ ] were found. Significant associations with the degree of albuminuria (normo- vs. micro- vs. macroalbuminuria) were observed for higher plasma levels of MMP-2 $\quad(\mathrm{p}$-trend $=0.028), \quad$ MMP-3 $\quad(\mathrm{p}$-trend $=0.004)$, MMP-10 (p-trend $=0.005)$ and TIMP-1 $(\mathrm{p}$-trend $=0.001)$ (Figure 2A).

\section{Associations between MMPs, TIMP-1 and retinopathy}

Proliferative retinopathy $(\mathrm{n}=146)$ was associated with higher levels of MMP-2 [0.71 (0.45; 0.96)], MMP-3 [0.49 $(0.28 ; 0.70)]$, MMP-10 [0.39 (0.11;0.66)] and TIMP-1

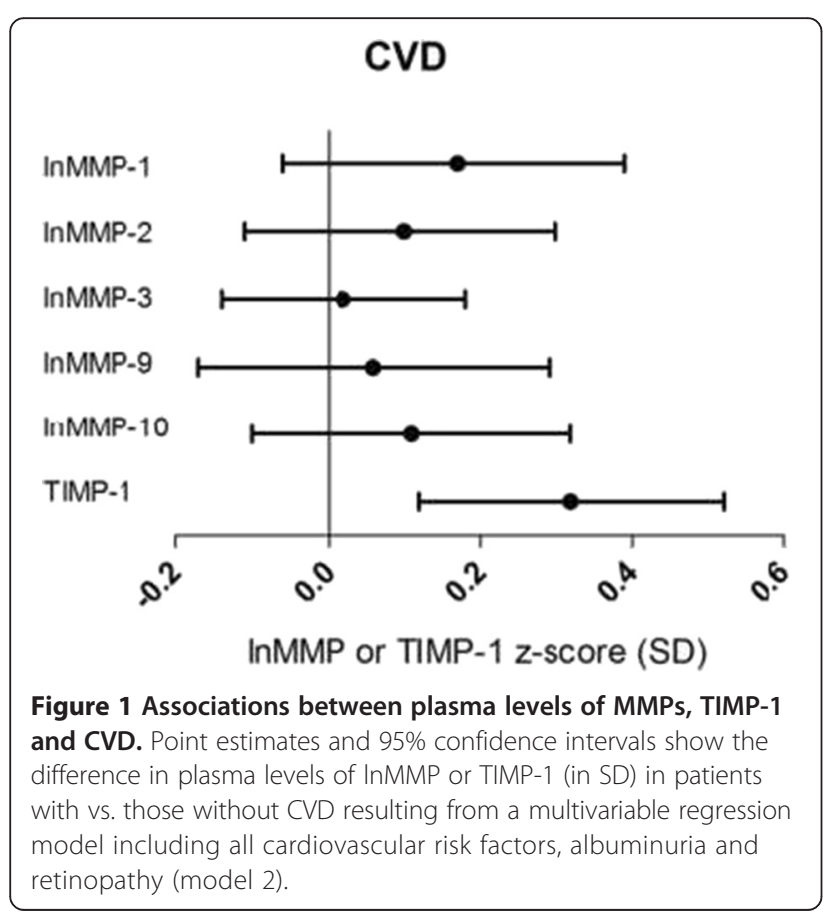

[0.54 $(0.28 ; 0.81)]$ after adjustment for age, sex, duration of diabetes and HbA1c (Additional file 3: Table S3, Model 1). However, after further adjustment for the other cardiovascular risk factors, CVD and albuminuria, only MMP-2 $[0.36(0.06 ; 0.66)]$ remained significantly higher in patients with proliferative retinopathy vs. those without retinopathy (Figure 2B; Additional file 3: Table S3, Model 2). In addition, a significant trend across the severity of retinopathy was observed for increasing plasma levels of MMP-2 ( $\mathrm{p}$-trend $=0.017$ ).

In contrast, non-proliferative retinopathy $(\mathrm{n}=125)$ was not significantly associated with higher plasma levels of MMPs or TIMP-1 (Figure 2B; Additional file 3: Table S3).

\section{Associations of MMPs and TIMP-1 with LGI and ED}

Associations between MMPs and TIMP-1 and the individual markers of LGI and ED are shown in Additional file 4: Table S4. After adjustment for all cardiovascular risk factors (model 2), MMP-1, $-3,-9,-10$, and TIMP1 , but not MMP-2, were positively and significantly associated with the LGI score. In addition, MMP-2, MMP-10 and TIMP-1 were positively and significantly associated with the ED score (Table 2, model 2). However, significant associations between plasma levels of MMPs and TIMP-1 on the one hand and CVD, albuminuria and retinopathy on the other hand were largely independent of LGI and ED, as these associations remained significant after adding the LGI and/or ED score to the model (Additional file 1: Table S1, Additional file 2: Table S2 and Additional file 3: Table S3; Models 3, 4 and 5).

\section{Additional analyses}

Adding use of ACE inhibitors specifically versus antihypertensive medication in general as covariable to our models, did not materially influence the results. Similarly, adjustment for urinary albumin excretion rate instead of albuminuria status and waist-hip-ratio instead of BMI did not change our results (data not shown). We have not included adjustment for TIMP-1 in model 2 in the main analyses between plasma levels of MMPs and vascular complications, because TIMP-1 levels are influenced by all MMPs and not just by one MMP. Therefore, we have also not analyzed associations between MMP/TIMP-1 ratios and vascular complications. However, additional adjustment in model 2 for TIMP-1 levels did also not materially influence our results.

In the relationship between MMPs, TIMP and vascular complications, eGFR (a marker of renal dysfunction) can be considered as a potential confounder or mediator. If eGFR should be a mediator rather than a confounder, models including eGFR may be overadjusted. We therefore reanalyzed the data without adjustment for eGFR; results were generally similar to models with such adjustment (data not shown). 


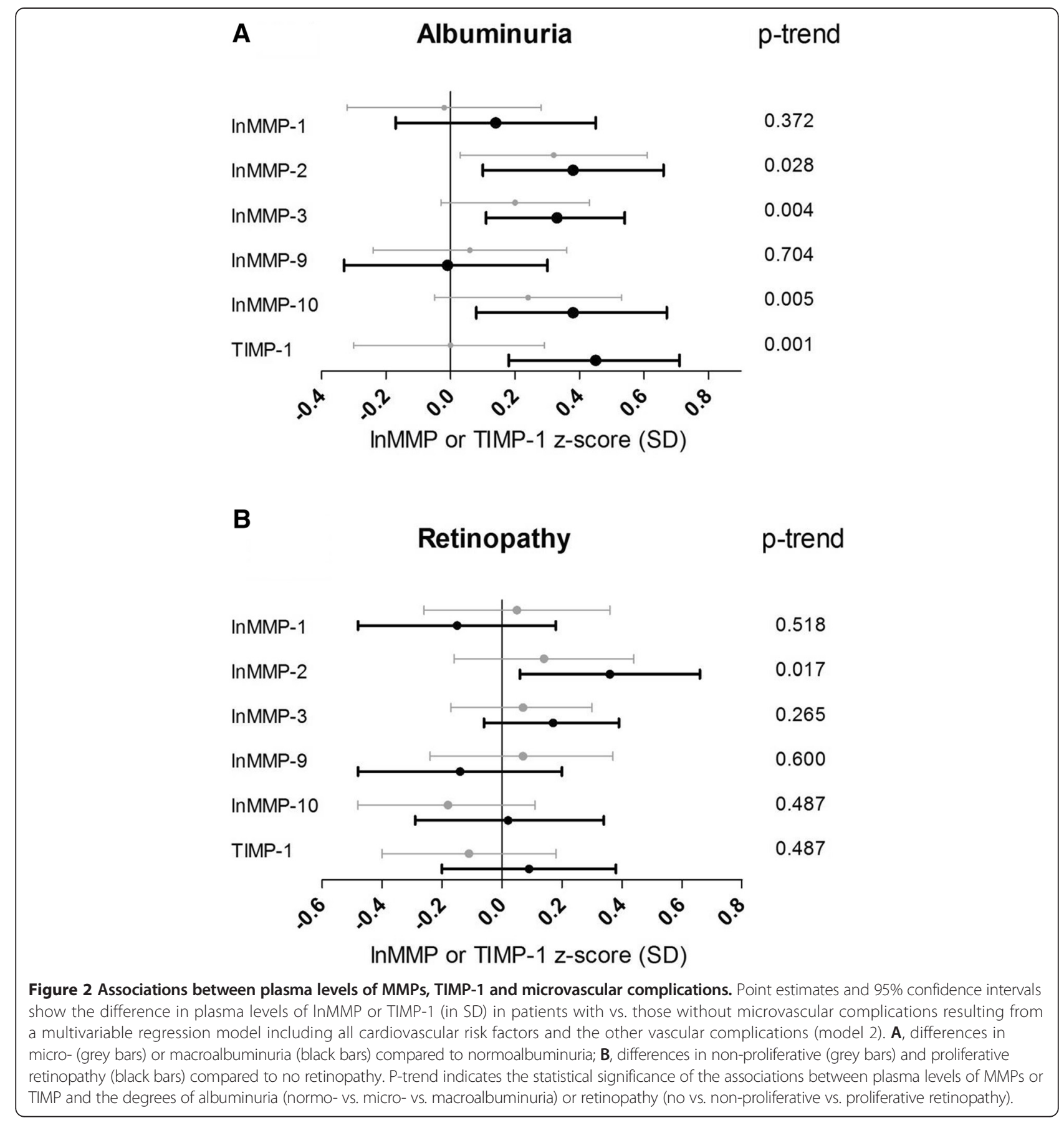

\section{Discussion}

This is the first study that comprehensively assessed associations of MMPs and TIMP-1 with long-term complications in type 1 diabetes. In a group of 493 patients with and without diabetes complications, we found that high plasma levels of TIMP-1 were associated with CVD. In addition, MMP-2, -3 and -10 , and TIMP-1 levels were associated with macroalbuminuria, and MMP-2 was associated with proliferative retinopathy. All these associations remained significant after adjustment for cardiovascular risk factors, prevalence of other vascular complications and markers of LGI and ED.

\section{MMPs, TIMP-1 and CVD}

Prevalent CVD was associated with higher plasma levels of TIMP-1, but not of MMP-1, $-2,-3,-9$ and -10 . In accordance, significantly higher levels of plasma TIMP-1 have been previously observed in two studies in type 2 diabetic patients with a history of coronary artery disease $[26,27]$. In addition, in a large prospective cohort study 
Table 2 Associations between InMMP-1, -2, -3, -9, -10 and TIMP-1 and the LGI and ED scores

\begin{tabular}{|c|c|c|c|c|c|c|c|}
\hline & \multirow[b]{2}{*}{ Model } & \multicolumn{3}{|c|}{ Inflammation (z-score) } & \multicolumn{3}{|c|}{ Endothelial dysfunction (z-score) } \\
\hline & & $\beta$ & $95 \% \mathrm{Cl}$ & p-value & $\beta$ & $95 \% \mathrm{Cl}$ & $\mathrm{p}$-value \\
\hline \multirow[t]{3}{*}{ MMP-1 } & 1 & 0.07 & $-0.02 ; 0.15$ & 0.112 & 0.00 & $-0.09 ; 0.09$ & 0.998 \\
\hline & 2 & 0.08 & $0.00 ; 0.16$ & 0.047 & 0.00 & $-0.09 ; 0.08$ & 0.987 \\
\hline & 3 & 0.08 & $-0.00 ; 0.15$ & 0.062 & -0.01 & $-0.09 ; 0.08$ & 0.834 \\
\hline \multirow[t]{3}{*}{ MMP-2 } & 1 & 0.10 & $0.01 ; 0.18$ & 0.032 & 0.19 & $0.10 ; 0.27$ & $<0.001$ \\
\hline & 2 & 0.03 & $-0.06 ; 0.12$ & 0.526 & 0.14 & $0.04 ; 0.23$ & 0.004 \\
\hline & 3 & 0.00 & $-0.08 ; 0.09$ & 0.950 & 0.13 & $0.04 ; 0.22$ & 0.006 \\
\hline \multirow[t]{3}{*}{ MMP-3 } & 1 & 0.26 & $0.16 ; 0.36$ & $<0.001$ & 0.08 & $-0.03 ; 0.19$ & 0.137 \\
\hline & 2 & 0.19 & $0.09 ; 0.30$ & 0.001 & -0.02 & $-0.14 ; 0.10$ & 0.715 \\
\hline & 3 & 0.17 & $0.06 ; 0.28$ & 0.003 & -0.04 & $-0.16 ; 0.08$ & 0.474 \\
\hline \multirow[t]{3}{*}{ MMP-9 } & 1 & 0.16 & $0.08 ; 0.25$ & $<0.001$ & -0.02 & $-0.11 ; 0.06$ & 0.592 \\
\hline & 2 & 0.17 & $0.09 ; 0.24$ & $<0.001$ & -0.02 & $-0.10 ; 0.07$ & 0.718 \\
\hline & 3 & 0.16 & $0.08 ; 0.24$ & $<0.001$ & -0.02 & $-0.10 ; 0.06$ & 0.577 \\
\hline \multirow[t]{3}{*}{ MMP-10 } & 1 & 0.24 & $0.16 ; 0.32$ & $<0.001$ & 0.13 & $0.04 ; 0.21$ & 0.003 \\
\hline & 2 & 0.22 & $0.14 ; 0.30$ & $<0.001$ & 0.11 & $0.02 ; 0.20$ & 0.019 \\
\hline & 3 & 0.21 & $0.13 ; 0.30$ & $<0.001$ & 0.10 & $0.01 ; 0.19$ & 0.029 \\
\hline \multirow[t]{3}{*}{ TIMP-1 } & 1 & 0.34 & $0.27 ; 0.43$ & $<0.001$ & 0.25 & $0.17 ; 0.33$ & $<0.001$ \\
\hline & 2 & 0.29 & $0.21 ; 0.38$ & $<0.001$ & 0.21 & $0.12 ; 0.30$ & $<0.001$ \\
\hline & 3 & 0.28 & $0.20 ; 0.37$ & $<0.001$ & 0.20 & $0.11 ; 0.29$ & $<0.001$ \\
\hline
\end{tabular}

$\beta$, standardized regression coefficient: indicates increase in the low-grade inflammation and endothelial dysfunction score (in SDs) per 1 SD increase in InMMP-1, $-2,-3,-9,-10$ or TIMP-1. Cl: confidence interval. MMP: matrix metalloproteinase.

Model 1: adjusted for age, sex, duration of diabetes and HbA1c.

Model 2: model $1+B M l$, triglycerides, LDL, HDL, systolic blood pressure, eGFR, smoking and antihypertensive medication.

Model 3: model $2+C V D$, albuminuria and retinopathy.

of individuals with and without (presumably type 2) diabetes, increased plasma TIMP-1 levels, measured six months after myocardial infarction, was significantly associated with left ventricular remodeling and adverse outcomes [28]. In the current study on individuals with type 1 diabetes, measurements of plasma TIMP-1 were, by its cross-sectional design, performed 1 month to 10 years after the cardiovascular event. Although we cannot prove a relationship between elevated plasma levels of TIMP-1 and new cardiovascular events, TIMP1 may be a marker for abnormal regulation of matrix remodeling after a cardiovascular event. This is supported by studies in animal models showing elevation of plasma TIMP-1 in type 1 diabetic minipigs with left ventricular hypertrophy, increased cardiac fibrosis and cardiac dysfunction [29].

\section{MMPs, TIMP-1 and albuminuria}

Plasma levels of MMP-2 were significantly higher in both micro- and macroalbuminuric patients compared to those with normoalbuminuria. In contrast, a previous study in type 1 diabetic patients did not show increased levels of MMP-2 in microalbuminuric vs. normoalbuminuric individuals [7]. This discrepancy may be explained by the very small sample size $(\mathrm{n}=12)$ of that study [7].
Thus far, no studies have been reported regarding plasma MMP-3 and diabetic nephropathy in humans or animals. Our study is the first study showing that higher plasma levels of MMP-3 are associated with increasing levels of albuminuria. In accordance with our study, in type 2 diabetic patients, high interstitial MMP-3 mRNA expression was associated with higher degrees of interstitial injury [30]. The increased plasma MMP-3 could therefore potentially originate from the renal interstitium. This finding may be biologically explained by the fact that type IV collagen, one of the major substrates of proteolysis by MMP-3, is an important component of the basal lamina of the glomerular basement membrane, Bowman's capsule, and of the basal membranes of renal tubules and vessels [31]. MMP-3-dependent type IV collagen proteolysis therefore constitutes a potential explanation for the current observation of a strong association between MMP-3 and albuminuria.

In addition, higher plasma MMP-10 levels were significantly associated with macroalbuminuria in our study. This result is in full agreement with a recent study [8] in patients with type 1 diabetes, in which a higher plasma level of MMP-10 was associated with diabetic nephropathy independently of classical risk factors. In addition, MMP-10 knockout (streptozotocin 
(STZ)-induced) diabetic mice showed lower renal mesangial cell expansion and lower renal macrophage influx compared to wildtype diabetic mice [8], suggesting that MMP-10 may be involved in the onset of diabetic nephropathy.

Besides MMP-2, -3, and -10, TIMP-1 was also associated with macroalbuminuria. This association was also observed in one-sided nephrectomized type 1 diabetic rats compared to non-diabetic controls [32]. These results reflect a possible role for TIMP-1 in increased renal fibrosis in early diabetic nephropathy.

\section{MMPs, TIMP-1 and retinopathy}

Plasma MMP-2 levels showed a significant positive association with proliferative retinopathy. Salzmann et al. [33] showed an overall increase of different MMPs $(-2$, -3 and -9$)$ and TIMP-1 in specimens of retinal tissue of patients with proliferative diabetic retinopathy compared to retinal tissue of patients without diabetes. Also, increased retinal levels of MMP-2 were found in rats and mice with STZ-induced diabetes compared to non-diabetic controls [34]. Our current clinical results, supported by the relatively scarce literature, indicate that MMP-2 may play a role the pathogenesis of proliferative diabetic retinopathy. In addition, the study by Toni et al. [8] has shown a significant association between plasma MMP-10 and proliferative retinopathy, which is in accordance with our current findings in the minimally adjusted model (including age, sex, duration of diabetes and HbA1c). However, this was no longer significant after additional adjustment for cardiovascular risk factors and the presence of CVD and albuminuria. Since Toni et al. did not adjust for the presence of other vascular complications, this could explain the observed difference between their and our study.

We did not find a significant association of plasma MMPs or TIMP-1 levels with non-proliferative retinopathy. This is in contrast with the results of the study of Jacqueminet et al. [10], in which increased serum MMP9 levels were shown in patients with type 1 diabetes with minimal to moderate retinopathy $(n=14)$ compared to diabetic patients without retinopathy and non-diabetic controls. Possibly the serum measurement does not truly reflect the circulating concentration of MMPs and TIMPs compared to the plasma measurement [35]. In addition, potential confounders may not have been fully addressed in this earlier study, but were shown to be of importance in the current study. In fact, also the current study showed a significant association between plasma MMP-9 levels and the presence of non-proliferative retinopathy in the crude model [standardized $\beta=0.22$ $(0.00 ; 0.44)]$, but this was attenuated after adjustment for confounding.
MMPs, TIMP-1, low-grade inflammation (LGI) and endothelial dysfunction (ED)

In our study, all MMPs, except MMP-2, were significantly associated with markers of low-grade inflammation (Table 2, model 2). Indeed, it is well known that MMP-2 has a more potent anti-inflammatory effect compared to other MMPs, which may be attributable to the fact that monocyte chemo-attractant protein-3 (MCP-3) is an important substrate of MMP-2. The product of MCP-3 after cleavage by MMP-2 antagonizes the effect of MCP-3 on the chemokine receptors-1, -2 and -3 and may thereby attenuate the inflammatory response [36]. Although MMP-1 and MMP-3 are known to cleave MCP-1, -2 and -4 , the antagonistic effects of the cleaved products on inflammation are minimal, because of lower affinity for the chemokine receptors [37]. MMPs can be activated and up-regulated by mediators of inflammation, but also have an intrinsic effect on the inflammatory response [38]. Although the exact role of MMPs in the inflammatory cascade remains incompletely understood, our results suggest that associations of MMPs and TIMP-1 with complications of type 1 diabetes are unlikely to be explained by LGI.

MMP-2, MMP-10 and TIMP-1 were associated with markers of ED. These findings are in line with literature demonstrating that MMP-2 is able to degrade occludin, a tight junction protein, in the blood retinal membrane leading to increased vascular permeability [12], and that MMP-10 can induce endothelial proteolysis in response to CRP [39]. In addition, plasma TIMP-1 levels are associated with endothelial cell migration [40]. Thus, associations between MMP and TIMP-1 levels and ED are biologically plausible; nevertheless, our results suggest that associations of MMPs and TIMP-1 with complications of type 1 diabetes are unlikely to be explained by ED.

\section{Limitations}

There are several limitations to our study. First, the cross-sectional design only allows speculations on causality. We thus cannot exclude that increased levels of plasma MMPs and TIMP-1 may be a result instead of a cause of vascular complications. Second, circulating levels of biomarkers may reflect specific organ injury, but can also originate from other tissues. We adjusted for other known vascular complications, but cannot rule out that other causative factors may contribute and the observed associations could therefore be underestimated. In addition, MMP- $1,-3,-10$ and TIMP-1 have a smaller molecular weight than albumin $[41,42]$ and may be filtered in the glomerulus, especially in albuminuric patients, which may also have underestimated the associations between these biomarkers and vascular complications. Third, plasma samples can be obtained easily 
and we thus used plasma levels to investigate the involvement of MMPs and TIMP-1 in the pathogenesis of vascular complications in type 1 diabetes. However, we do not know whether plasma markers truly reflect the local pathological situation at the tissue level. Finally, our results suggest that associations of MMPs and TIMP-1 with complications of type 1 diabetes are unlikely to be explained by LGI or ED, but we cannot exclude roles of LGI or ED not reflected by the biomarkers we used.

\section{Conclusion}

In this study with type 1 diabetic patients, plasma MMP-2, MMP-3, MMP-10 and TIMP-1 levels are associated with macro- and microvascular complications and these associations were largely independent of LGI and ED. Further (prospective) studies are warranted to elucidate the involvement of MMPs and TIMPs in the development of diabetic macro- and microvascular complications.

\section{Additional files}

Additional file 1: Table S1. Associations between InMMP-1, InMMP2, InMMP-3, InMMP-9, InMMP-10 and TIMP-1 and cardiovascular disease.

Additional file 2: Table S2. Associations between InMMP-1, InMMP-2, InMMP-3, InMMP-9, InMMP-10 and TIMP-1 and microalbuminuria or macroalbuminuria.

Additional file 3: Table S3. Associations between InMMP-1, InMMP-2, InMMP-3, InMMP-9, InMMP-10 and TIMP-1 and non-proliferative or proliferative retinopathy.

Additional file 4: Table S4. Associations between plasma levels of MMP-1, $-2,-3,-9$, and -10 and TIMP-1 and markers of low-grade inflammation and endothelial dysfunction.

\section{Abbreviations \\ CVD: Cardiovascular disease; ED: Endothelial dysfunction; LGl: Low-grade inflammation; MMP: Matrix metalloproteinase; TIMP: Tissue inhibitor of metalloproteinase}

\section{Competing interests}

The authors declare that they have no competing interests.

\section{Authors' contributions}

S.P. and L.E. researched the data and drafted the article; S.P., L.E., C.G.S. and C. D.S. wrote the manuscript. J.B., N.C., J.F. reviewed and edited the manuscript for important intellectual content. N.C. and J.F. led the grants which supported the study. C.G.S. and C.D.S. had equal contributions, contributed to the discussion and reviewed and edited the manuscript for important intellectual content. All authors read and approved the final manuscript.

\section{Acknowledgements}

We would like to thank all the investigators and participants who very kindly participated in the study. Members of the EURODIAB PCS group are B. Karamanos, A. Kofinis, K. Petrou, Hippokration Hospital, Athens, Greece; F. Giorgino, G. Picca, A. Angarano, G. de Pergola, L. Laviola, R. Giorgino, Internal Medicine, Endocrinology and Metabolic Diseases, Department of Emergency and Organ Transplantation, University of Bari, Bari, Italy; C. Ionescu-Tirgoviste, A. Coszma, C. Guja, Clinic of Diabetes, Nutrition and Metabolic Diseases, Bucharest, Romania; M. Songini, A. Casu, M. Pedron, S. Pintus, M. Fossarello, Diabetes Unit Ospedale San Michele, Cagliari, Italy; J. B. Ferriss, G. Grealy, D. O'Keefe, Cork University Hospital, Cork, Ireland; M. Toeller, C. Arden, Diabetes Research Institute, Heinrich-Heine University, Duesseldorf, Germany; R. Rottiers, C. Tuyttens, H. Priem, University Hospital of Gent, Belgium; P. Ebeling, M. Kylliäinen, V. A. Koivisto, University Hospital of Helsinki, Finland; B. Idzior-Walus, J. Sieradzki, K.
Cyganek, B. Solnica, Department of Metabolic Diseases, Jagiellonian University, Krakow, Poland; H. H. P. J. Lemkes, J. C. Lemkes-Stuffken, Leiden University Medical Centre, the Netherlands; J. Nunes-Correa, M. C. Rogado, L. Gardete-Correia, M. C. Cardoso, A. Silva, J. Boavida, M. Machado Sa Marques, Portuguese Diabetic Association, Lisbon, Portugal; G. Michel, R. Wirion, S. Cardillo, Centre Hospitalier, Luxembourg; G. Pozza, R. Mangili, V. Asnaghi, Ospedale San Raffaele, Milan, Italy; E. Standl, B. Schaffler, H. Brand, A. Harms, City Hospital Schwabing, Munich, Germany; D. Ben Soussan, O. Verier-Mine, P. Fallas, M. C. Fallas, Centre Hospitalier de Valenciennes, France; J. H. Fuller, J. Holloway, L. Asbury, D. J. Betteridge, University College London, UK; G. Cathelineau, A. Bouallouche, B. Villatte Cathelineau, Hospital Saint-Louis, Paris, France; F. Santeusanio, G. Rosi, V. D'Alessandro, C. Cagini, P. Bottini, G. P. Reboldi, Dipartimento di Medicina Interna, Perugia, Italy; R. Navalesi, G. Penno, S. Bandinelli, R. Miccoli, M. Nannipieri, Dipartimento di Endocrinologia e Metabolismo, Pisa, Italy; G. Ghirlanda, C. Saponara, P. Cotroneo, A. Manto, A. Minnella, Universita Cattolica del Sacro Cuore, Rome, Italy; J. D. Ward, S. Tesfaye, S. Eaton, C. Mody, Royal Hallamshire Hospital, Sheffield, UK; M. Borra, P. Cavallo Perin, S. Giunti, G. Grassi, G. F. Pagano, M. Porta, R. Sivieri, F. Vitelli, M. Veglio, Dipartimento di Medicina Interna, Università di Torino and ASO TO/CRF/Maria Adelaide, Turin, Italy; N. Papazoglou, G. Manes, General Hospital Papageorgiou, Diabetes Unit, Thessaloniki, Greece; M. Muggeo, M. lagulli, V. Cacciatori, V. Cattedra di Malattie del Metabolismo, Verona, Italy; K. Irsigler, H. Abrahamian, Hospital Vienna Lainz, Austria; S. Walford, J. Sinclair, S. Hughes, V. McLelland, J. Ward, New Cross Hospital, Wolverhampton, UK; G. Roglic, Z. Metelko, Z. R. Pepeonik, Vuk Vrhovac Institute for Diabetes, Zagreb, Croatia.

\section{Steering committee members}

J. H. Fuller (London), B. Karamanos, Chairman (Athens), A.-K. Sjolie (Odense), N. Chaturvedi (London), M. Toeller (Duesseldorf), G. Pozza Co-chairman (Milan), B. Ferriss (Cork), M. Porta (Turin), R. Rottiers (Gent), G. Michel (Luxembourg)

\section{Co-ordinating centre}

J. H. Fuller, N. Chaturvedi, J. Holloway, D. Webb, L. Asbury, University College London, UK

\section{Central laboratories}

G.-C. Viberti, R. Swaminathan, P. Lumb, A. Collins, S. Sankaralingham, M.A. Crook, Guy's and St Thomas Hospital, London, UK

\section{Retinopathy Grading Centre}

S. Aldington, T. Mortemore, H. Lipinski, Royal Postgraduate Medical School of Imperial College London, London, UK

\section{Nutrition Co-ordinating Centre}

M. Toeller, W.A. Scherbaum, F.A. Gries, Heinrich-Heine-University, Diabetes Research Institute and Department of Endocrinology, Diabetology and Rheumatology, Duesseldorf, Germany

The EURODIAB Prospective Complications Study was supported by grants from the Wellcome Trust, the European Community and Diabetes UK.

\section{Author details}

'Department of Internal Medicine, Maastricht University Medical Centre, P.O. Box 5800, 6202 AZ, Maastricht, the Netherlands. ²Department of Internal Medicine, Atrium Medical Centre, Heerlen, the Netherlands. ${ }^{3}$ Institute of Cardiovascular Sciences, University College London, London, UK. ${ }^{4}$ Department of Epidemiology and Public Health, University College London, London, UK.

Received: 13 October 2014 Accepted: 22 February 2015

Published online: 10 March 2015

\section{References}

1. Laing SP, Swerdlow AJ, Slater SD, Burden AC, Morris A, Waugh NR, et al. Mortality from heart disease in a cohort of 23,000 patients with insulin-treated diabetes. Diabetologia. 2003;46:760-5.

2. Soedamah-Muthu SS, Fuller JH, Mulnier HE, Raleigh VS, Lawrenson RA, Colhoun HM. High risk of cardiovascular disease in patients with type 1 diabetes in the U.K.: a cohort study using the general practice research database. Diabetes Care. 2006;29:798-804. 
3. Snell-Bergeon JK, Nadeau K. Cardiovascular disease risk in young people with type 1 diabetes. J Cardiovasc Transl Res. 2012;5:446-62.

4. Galis ZS, Khatri JJ. Matrix metalloproteinases in vascular remodeling and atherogenesis: the good, the bad, and the ugly. Circ Res. 2002;90:251-62.

5. Kadoglou NP, Daskalopoulou SS, Perrea D, Liapis CD. Matrix metalloproteinases and diabetic vascular complications. Angiology. 2005:56:173-89.

6. Visse R, Nagase H. Matrix metalloproteinases and tissue inhibitors of metalloproteinases: structure, function, and biochemistry. Circ Res. 2003:92:827-39.

7. Thrailkill KM, Bunn RC, Moreau CS, Cockrell GE, Simpson PM, Coleman HN, et al. Matrix metalloproteinase-2 dysregulation in type 1 diabetes. Diabetes Care. 2007;30:2321-6.

8. Toni M, Hermida J, Goni MJ, Fernandez P, Parks WC, Toledo E, et al. Matrix metalloproteinase-10 plays an active role in microvascular complications in type 1 diabetic patients. Diabetologia. 2013;56:2743-52.

9. Gharagozlian S, Svennevig K, Bangstad HJ, Winberg JO, Kolset SO. Matrix metalloproteinases in subjects with type 1 diabetes. BMC Clin Pathol. 2009;9:7.

10. Jacqueminet S, Ben Abdesselam O, Chapman MJ, Nicolay N, Foglietti MJ, Grimaldi A, et al. Elevated circulating levels of matrix metalloproteinase-9 in type 1 diabetic patients with and without retinopathy. Clin Chim Acta. 2006:367:103-7.

11. Thrailkill KM, Moreau CS, Cockrell GE, Jo CH, Bunn RC, Morales-Pozzo AE, et al. Disease and gender-specific dysregulation of NGAL and MMP-9 in type 1 diabetes mellitus. Endocrine. 2010;37:336-43.

12. Giebel SJ, Menicucci G, McGuire PG, Das A. Matrix metalloproteinases in early diabetic retinopathy and their role in alteration of the blood-retinal barrier. Lab Invest. 2005;85:597-607.

13. Soedamah-Muthu SS, Chaturvedi N, Schalkwijk CG, Stehouwer CD, Ebeling P. Fuller JH. Soluble vascular cell adhesion molecule-1 and soluble E-selectin are associated with micro- and macrovascular complications in Type 1 diabetic patients. J Diabetes Complication. 2006;20:188-95.

14. Costacou T, Lopes-Virella MF, Zgibor JC, Virella G, Otvos J, Walsh M, et al Markers of endothelial dysfunction in the prediction of coronary artery disease in type 1 diabetesThe Pittsburgh epidemiology of diabetes complications study. J Diabetes Complications. 2005;19:183-93.

15. Devaraj S, Cheung AT, Jialal I, Griffen SC, Nguyen D, Glaser N, et al. Evidence of increased inflammation and microcirculatory abnormalities in patients with type 1 diabetes and their role in microvascular complications. Diabetes. 2007;56:2790-6.

16. Chaturvedi N, Sjoelie AK, Porta M, Aldington SJ, Fuller JH, Songini M, et al. Markers of insulin resistance are strong risk factors for retinopathy incidence in type 1 diabetes. Diabetes Care. 2001;24:284-9.

17. Chaturvedi N, Bandinelli S, Mangili R, Penno G, Rottiers RE, Fuller JH. Microalbuminuria in type 1 diabetes: rates, risk factors and glycemic threshold. Kidney Int. 2001;60:219-27.

18. Schram MT, Chaturvedi N, Schalkwijk C, Giorgino F, Ebeling P, Fuller JH, et al. Vascular risk factors and markers of endothelial function as determinants of inflammatory markers in type 1 diabetes: the EURODIAB prospective complications study. Diabetes Care. 2003;26:2165-73.

19. Koivisto VA, Stevens LK, Mattock M, Ebeling P, Muggeo M, Stephenson J, et al. Cardiovascular disease and its risk factors in IDDM in Europe. EURODIAB IDDM complications study group. Diabetes Care. 1996;19:689-97.

20. Levey AS, Stevens LA, Schmid CH, Zhang YL, Castro 3rd AF, Feldman HI, et al. A new equation to estimate glomerular filtration rate. Ann Intern Med. 2009;150:604-12

21. Aldington SJ, Kohner EM, Meuer S, Klein R, Sjolie AK. Methodology for retinal photography and assessment of diabetic retinopathy: the EURODIAB IDDM complications study. Diabetologia. 1995;38:437-44.

22. Fossati $P$, Prencipe $L$. Serum triglycerides determined colorimetrically with an enzyme that produces hydrogen peroxide. Clin Chem. 1982;28:2077-80.

23. Sugiuchi H, Uji Y, Okabe H, Irie T, Uekama K, Kayahara N, et al. Direct measurement of high-density lipoprotein cholesterol in serum with polyethylene glycol-modified enzymes and sulfated alpha-cyclodextrin. Clin Chem. 1995:41:717-23.

24. Friedewald WT, Levy RI, Fredrickson DS. Estimation of the concentration of low-density lipoprotein cholesterol in plasma, without use of the preparative ultracentrifuge. Clin Chem. 1972;18:499-502.

25. Nin JW, Ferreira I, Schalkwijk CG, Prins MH, Chaturvedi N, Fuller JH, et al. Levels of soluble receptor for AGE are cross-sectionally associated with cardiovascular disease in type 1 diabetes, and this association is partially mediated by endothelial and renal dysfunction and by low-grade inflammation: the EURODIAB prospective complications study. Diabetologia. 2009:52:705-14

26. Opstad TB, Pettersen AA, Weiss TW, Akra S, Ovstebo R, Arnesen H, et al. Genetic variation, gene-expression and circulating levels of matrix metalloproteinase- 9 in patients with stable coronary artery disease. Clin Chim Acta. 2012;413:113-20.

27. Derosa G, Cicero AF, Scalise F, Avanzini MA, Tinelli C, Peros E, et al. Metalloproteinases in diabetics and nondiabetics during acute coronary syndromes and after 3 months. Endothelium. 2007;14:175-83.

28. Kelly D, Khan SQ, Thompson M, Cockerill G, Ng LL, Samani N, et al. Plasma tissue inhibitor of metalloproteinase-1 and matrix metalloproteinase-9: novel indicators of left ventricular remodelling and prognosis after acute myocardial infarction. Eur Heart J. 2008:29:2116-24.

29. Lu L, Zhang Q, Pu L, Peng WH, Yan XX, Wang $\sqcup$, et al. Dysregulation of matrix metalloproteinases and their tissue inhibitors is related to abnormality of left ventricular geometry and function in streptozotocin-induced diabetic minipigs. Int J Exp Pathol. 2008:89:125-37.

30. Suzuki D, Miyazaki M, Jinde K, Koji T, Yagame M, Endoh M, et al. In situ hybridization studies of matrix metalloproteinase-3, tissue inhibitor of metalloproteinase-1 and type IV collagen in diabetic nephropathy. Kidney Int. 1997:52:111-9.

31. Hosoyamada Y, Sakai T. Structural arrangement of collagen fibrils in the periarterial connective tissue of the kidney: their functional relevance as a structural stabilizer against arterial pressure. Anat Sci Int. 2012;87:80-7.

32. Alter ML, Kretschmer A, Von Websky K, Tsuprykov O, Reichetzeder C, Simon $A$, et al. Early urinary and plasma biomarkers for experimental diabetic nephropathy. Clin Lab. 2012;58:659-71.

33. Salzmann J, Limb GA, Khaw PT, Gregor ZJ, Webster L, Chignell AH, et al. Matrix metalloproteinases and their natural inhibitors in fibrovascular membranes of proliferative diabetic retinopathy. Br J Ophthalmol. 2000;84:1091-6.

34. Mohammad G, Kowluru RA. Matrix metalloproteinase-2 in the development of diabetic retinopathy and mitochondrial dysfunction. Lab Invest. 2010:90:1365-72.

35. Jung K, Gerlach RF, Tanus-Santos JE. Preanalytical pitfalls of blood sampling to measure true circulating matrix metalloproteinase 9 and tissue inhibitors of matrix metalloproteinases. Clin Chim Acta. 2006;373:180-1. author reply 182.

36. McQuibban GA, Gong JH, Tam EM, McCulloch CA, Clark-Lewis I, Overall CM. Inflammation dampened by gelatinase a cleavage of monocyte chemoattractant protein-3. Science. 2000;289:1202-6.

37. McQuibban GA, Gong JH, Wong JP, Wallace JL, Clark-Lewis I, Overall CM. Matrix metalloproteinase processing of monocyte chemoattractant proteins generates CC chemokine receptor antagonists with anti-inflammatory properties in vivo. Blood. 2002;100:1160-7

38. Chase AJ, Newby AC. Regulation of matrix metalloproteinase (matrixin) genes in blood vessels: a multi-step recruitment model for pathological remodelling. J Vasc Res. 2003;40:329-43.

39. Montero I, Orbe J, Varo N, Beloqui O, Monreal Jl, Rodriguez JA, et al. C-reactive protein induces matrix metalloproteinase-1 and -10 in human endothelial cells: implications for clinical and subclinical atherosclerosis. J Am Coll Cardiol. 2006:47:1369-78.

40. Akahane T, Akahane M, Shah A, Connor CM, Thorgeirsson UP. TIMP-1 inhibits microvascular endothelial cell migration by MMP-dependent and MMP-independent mechanisms. Exp Cell Res. 2004:301:158-67.

41. Raffetto JD, Khalil RA. Matrix metalloproteinases and their inhibitors in vascular remodeling and vascular disease. Biochem Pharmacol. 2008;75:346-59.

42. Brew $\mathrm{K}$, Nagase $\mathrm{H}$. The tissue inhibitors of metalloproteinases (TIMPs): an ancient family with structural and functional diversity. Biochim Biophys Acta. 1803:2010:55-71. 